\title{
Compact Electro-Permeabilization System for Controlled Treatment of Biological Cells and Cell Medium Conductivity Change Measurement
}

\author{
Vitalij Novickij ${ }^{1}$, Audrius Grainys ${ }^{1}$, Jurij Novickij ${ }^{1}$, Sonata Tolvaisiene ${ }^{1}$, Svetlana Markovskaja ${ }^{2}$ \\ ${ }^{1}$ High Magnetic Field Institute, Vilnius Gediminas Technical University, Naugarduko 41, 03227, Vilnius, Lithuania, \\ vitalij.novickij@vgtu.lt \\ ${ }^{2}$ Laboratory of Mycology, Institute of Botany of Nature Research Centre, Zaliuju ezeru 49, 08406, Vilnius, Lithuania, \\ svetlana.markovskaja@botanika.lt
}

\begin{abstract}
Subjection of biological cells to high intensity pulsed electric field results in the permeabilization of the cell membrane. Measurement of the electrical conductivity change allows an analysis of the dynamics of the process, determination of the permeabilization thresholds, and ion efflux influence. In this work a compact electro-permeabilization system for controlled treatment of biological cells is presented. The system is capable of delivering $5 \mu \mathrm{s}-5 \mathrm{~ms}$ repetitive square wave electric field pulses with amplitude up to $1 \mathrm{kV}$. Evaluation of the cell medium conductivity change is implemented in the setup, allowing indirect measurement of the ion concentration changes occurring due to the cell membrane permeabilization. The simulation model using SPICE and the experimental data of the proposed system are presented in this work. Experimental data with biological cells is also overviewed.
\end{abstract}

Keywords: Electroporation, electric field, MOSFET, medium conductivity.

\section{INTRODUCTION}

$\mathrm{I}_{\mathrm{a}}^{\mathrm{N}}$ $N$ THE PAST decades investigation of the effects of the electric fields on the living cells and microorganisms has led to discovery of various cell polarization phenomena, which have led to modern biomedical techniques such as electroporation or dielectrophoresis [1]-[4]. Electroporation or electro-permeabilization is a popular biomedical technique, which is used for gene transfection, cell hybridization, drug delivery or cell lysis [5], [6]. The effect is dependent on the shape of the pulse, electric field amplitude, the number of pulses, and the geometry of the electrodes [7].

During electroporation the membrane of living cells is damaged and depending on the treatment intensity the effect could be reversible or irreversible [8]-[11]. In both cases the treatment processes act on a molecular level. In reversible or irreversible electroporation the treatment results in the change of the ion concentration of the cell medium. Such phenomenon occurs due to the release of the ions through the nanoscale pores that are caused by the high intensity electric field [8]-[11]. The real-time measurement of the medium conductivity allows the acquisition of additional information about the treatment intensity and the dynamics of the electroporation process, therefore, it is desirable in this field [12], [13]. By means of measurement of the change of the electric conductivity the extent of the permeabilization process can be evaluated. Also Pavlin et al. [14] have shown that the changes in conductivity occur due to the ion efflux in low-conductive mediums and colloidosmotic swelling in both the high and low-conductive mediums. By measuring the electric conductivity the limit permeabilization threshold can be determined. However, the measurement during the pulses should be implemented, while the conductivity measurement seconds after the pulsing procedures is not applicable [14], [15]. Various methods for the conductivity measurement of the electroporated tissue or cells were introduced in the last decade. The most popular techniques, electrical impedance tomography and magnetic resonance imaging, are used to measure conductivity of the electroporated tissue [16]-[20]. These methods examine the outcomes of the treatment procedure. For real-time or active observation of the treatment intensity the voltage or current measurements of the treated cells can be applied [21]. The real-time affected cell conductivity measurement can give ability to monitor the treated cells and introduce a feedback for the control of the treatment intensity in real time. Currently, separate RCL measurement devices connected to personal computer or data storage are applied [21], [22]. Such configurations involve additional complexity in the experimental setups, synchronization problems arise and the price of the whole biomedical system is increased.

Due to the many applications of the electroporation technique the development of the high power instrumentation applicable in this field is performed. Square wave electrical pulse systems are offering precise treatment intensity control due to the short rise and fall times and the pulse plateau region, and therefore are preferable in electroporation [23]-[25].

Due to the variety of the cell types and cell buffers used in biological sciences, the load of the electroporation system varies in a broad range (from several tens of $\Omega$ up to several $\mathrm{k} \Omega$ ). Therefore, high current handling capability (>30 A) must be ensured, which creates challenges to form repetitive pulses due to the capacitor discharge. As a rule, trade-offs between the generating capabilities, the load handling and the size of the facility are made. 
This work presents a square wave pulsed electric field system based on a MOSFET that offers high current handling capability ( $>50 \mathrm{~A}$ ) across the $5 \mu \mathrm{s}-5 \mathrm{~ms}$ range of the generated pulses. The novelty of the system is also based on the capability of electric conductivity change measurement during the pulsing.

\section{THEORY}

During the electroporation the transmembrane potential of the cell is altered, which results in the appearance of pores in the membrane and the increase of the ion transfer [26], [27]. The higher ion concentration in the cell medium results in the increase of the total medium electrical conductivity.

The electrical conductivity of the cell medium could be evaluated by the application of the Maxwell equation as a mean-field approximation. However, the calculation is complex due to the interaction between the cells, therefore, as a rule, the averaged approximate solutions are used. According to Maxwell, and as recently summarized by Pavlin et al., the effective conductivity $\sigma$ of a solution could be approximated as [14]:

$$
\frac{\sigma_{m}-\sigma}{2 \sigma_{m}+\sigma}=f \frac{\sigma_{m}-\sigma_{p}}{2 \sigma_{m}+\sigma_{p}},
$$

where $\sigma_{m}$ - the electrical conductivity of the medium, $\sigma_{p}-$ the conductivity of the particle and $f=\frac{n V_{\text {cell }}}{V_{m}}, n$ - number of cells, $V_{\text {cell }}$ - volume of single cell, $V_{m}$ - volume of the cell medium.

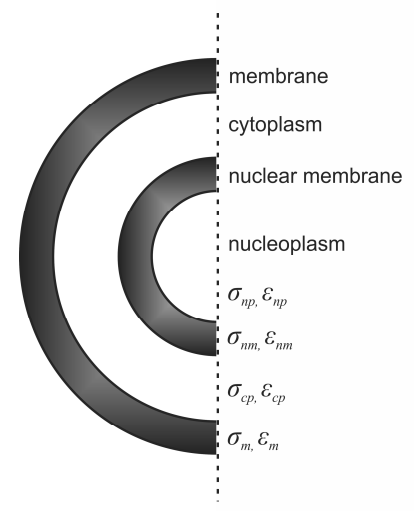

Fig. 1. Model of a biological cell.

However, further approximations are required, because the biological cell is not homogeneous. The lipid membrane has a low electrical conductivity $\sigma_{m b}$, while the interior is an electrolyte with relatively high conductivity $\sigma_{i n}$. According to Polevaya et al. the equivalent conductivity is derived [28]:

$$
\sigma_{e q}=\sigma_{m b} \frac{2(1-v) \sigma_{m b}+(1+2 v) \sigma_{i n}}{(2+v) \sigma_{m b}+(1-v) \sigma_{i n}}
$$

where $v=\left(1-\frac{d}{r}\right)^{3}, d-$ cell membrane thickness, $r-$ cell radius

For cells with a nucleus the electrical parameters of the nuclear membrane and the nucleoplasm must be taken into account. The model of such a cell is presented in Fig.1.

The $\sigma_{c p}, \sigma_{n m}, \sigma_{n p}, \varepsilon_{c p}, \varepsilon_{n m}, \varepsilon_{n p}$ are the electrical conductivity and permittivity values of the cytoplasm, the nuclear membrane and the nucleoplasm, respectively.

The solution becomes more complex and can be approximated as [29]:

$$
\begin{gathered}
\sigma_{e q} \approx \sigma_{c p} \frac{2(1-v)+\frac{\varepsilon_{n p}}{\varepsilon_{c p}}(1+2 v)}{(2+v)+(1-v) \frac{\varepsilon_{n p}}{\varepsilon_{c p}}}- \\
-\left(\begin{array}{c}
\left.\frac{\varepsilon_{n p} \sigma_{c p}-\varepsilon_{c p} \sigma_{n p}}{\varepsilon_{c p}} \cdot \frac{9 v}{\left[(2+v)+(1-v) \frac{\varepsilon_{n p}}{\varepsilon_{c p}}\right.}\right]^{2}
\end{array} .\right.
\end{gathered}
$$

The electrical conductance $G$ is a function of both physical parameters of the conductor and the conductivity of the material:

$$
G=\frac{\sigma A}{l}
$$

where $A$ - the conductor cross-sectional area and $l-$ length. During the electric field treatment the cuvette is stationary and the volume of the electrolyte is constant. Thus, the assumption that $\Delta G=\Delta \sigma$ can be made. The assumption is not true when the exposure or the waiting time between the pulses is long due to the evaporation processes in the cuvette, however, it is not applicable in this case.

\section{EVALUATION OF SYSTEM PARAMETERS}

The load of the electroporation system is an electrolytic buffer with biological cells. As it has been mentioned above, the impedance of the resulting electrolyte can vary in a broad range (from several tens of $\Omega$ up to several $\mathrm{k} \Omega$ ) depending on the buffer and the type of the cells that are used. In order to ensure the high applicability of the device, the whole range of loads must be covered, which requires high current application. The summary of the required system parameters is presented in Table 1 .

Table 1. Summary of the required system parameters.

\begin{tabular}{|c|c|c|}
\hline Parameter & Value & Denotation \\
\hline Voltage range & $0-1 \mathrm{kV}$ & $\mathrm{U}$ \\
\hline Pulse width & $5 \mu \mathrm{s}-5 \mathrm{~ms}$ & $\mathrm{t}_{\mathrm{P}}$ \\
\hline $\begin{array}{c}\text { Capacitor } \\
\text { discharge during } \\
\text { single pulse }\end{array}$ & $<25 \%$ & $\Delta \mathrm{U}_{\mathrm{MAX}}$ \\
\hline Pulse frequency & $0.1-100 \mathrm{~Hz}$ & $\mathrm{f}$ \\
\hline Maximum load & $20 \Omega$ & $\mathrm{R}_{\mathrm{MIN}}$ \\
\hline Peak current & $50 \mathrm{~A}$ & $\mathrm{I}_{\text {Peak }}$ \\
\hline
\end{tabular}


High currents result in a rapid discharge of the capacitor array. The increase of the total capacitance will inevitably increase the dimensions of the device, which is undesirable. We assumed that in order to generate an approximated square-wave electrical pulse, the discharge of the capacitor must not exceed $25 \%$ at the marginal case (maximum load of $20 \Omega$ during a single maximum width pulse of $5 \mathrm{~ms}$ ). The assumption has been made based on the parameters of the commercially available electroporators and the square wave electroporation protocols. As a result, the whole array of the required loads will be covered. It should be noted that in the majority of the electroporation experiments the resistance is higher than $20 \Omega$, therefore, the discharge will be even lower.

As a switch for the electrical pulse generating we decided to use a MOSFET to ensure low losses during the switching and the low rise and fall times.

As it has been mentioned above, the cell buffer impedance should be introduced in the setup for the real-time treatment analysis. Also the system must have a repetitive pulsing option for the short pulses with controllable frequency.

In order to develop a pulsed power system with the described parameters several trade-offs between the maximum capacitance, peak current, maximum load and the dimensions of the final setup must be made. In order to achieve the best result a SPICE simulation of the system has been introduced.

\section{EVALUATION OF SYSTEM PARAMETERS}

The parameters of the MOSFET model in the SPICE simulation have been adjusted to match all of the discussed requirements of the electroporation system (pulse rise/fall times, peak current, etc.). Based on the received characteristics a commercial MOSFET suitable for the electroporation system can be chosen. The summary of the developed MOSFET model is presented in Table 2.

Table 2. Summary of MOSFET SPICE model parameters.

\begin{tabular}{|c|c|c|}
\hline Parameter & Value & Denotation \\
\hline Drain-source voltage & $1.1 \mathrm{kV}$ & $\mathrm{V}_{\mathrm{DS}}$ \\
\hline $\begin{array}{c}\text { On-state resistance } \\
\text { current }\end{array}$ & $0.4 \Omega$ & $\mathrm{R}_{\mathrm{DS}}$ \\
\hline $\begin{array}{c}\text { Continuous drain } \\
\text { Pulsed drain current }\end{array}$ & $15 \mathrm{~A}$ & $\mathrm{I}_{\mathrm{D}}$ \\
\hline Gate charge & $180 \mathrm{nC}$ & $\mathrm{I}_{\mathrm{DP}}$ \\
\hline $\begin{array}{c}\text { Zero bias threshold } \\
\text { voltage }\end{array}$ & $5.4 \mathrm{~V}$ & $\mathrm{Q}_{\mathrm{G}}$ \\
\hline $\begin{array}{c}\text { Channel length } \\
\text { modulation parameter }\end{array}$ & $0.03 \mathrm{~V}^{-1}$ & $\mathrm{Lambda}$ \\
\hline $\begin{array}{c}\text { G-to-D capacitance } \\
\text { G-to-S capacitance }\end{array}$ & $9 \mathrm{nF}$ & $\mathrm{C}_{\mathrm{GD}}$ \\
\hline $\begin{array}{c}\text { Junction oxide } \\
\text { capacitance }\end{array}$ & $12 \mathrm{nF}$ & $\mathrm{C}_{\mathrm{GS}}$ \\
\hline $\begin{array}{c}\text { Junction grading } \\
\text { coefficient }\end{array}$ & 0.6 & $\mathrm{C}_{\mathrm{JO}}$ \\
\hline Junction voltage drop & $0.7 \mathrm{~V}$ & $\mathrm{~V}_{\mathrm{J}}$ \\
\hline D/S leakage current & $10 \mathrm{pA}$ & $\mathrm{I}_{\mathrm{S}}$ \\
\hline $\begin{array}{c}\text { Transconductance } \\
\text { parameter }\end{array}$ & 650 & $\mathrm{~K}_{\mathrm{P}}$ \\
\hline
\end{tabular}

The developed model allows the acquisition of the nanosecond range rise and fall times of the square wave electrical pulses with a $20 \Omega$ load $(1 \mathrm{kV} \mathrm{V}$ DS $)$, which is in compliance with the discussed system requirements. The model has been also tested with different loads and capacitor values. The results are presented in Fig. 2.
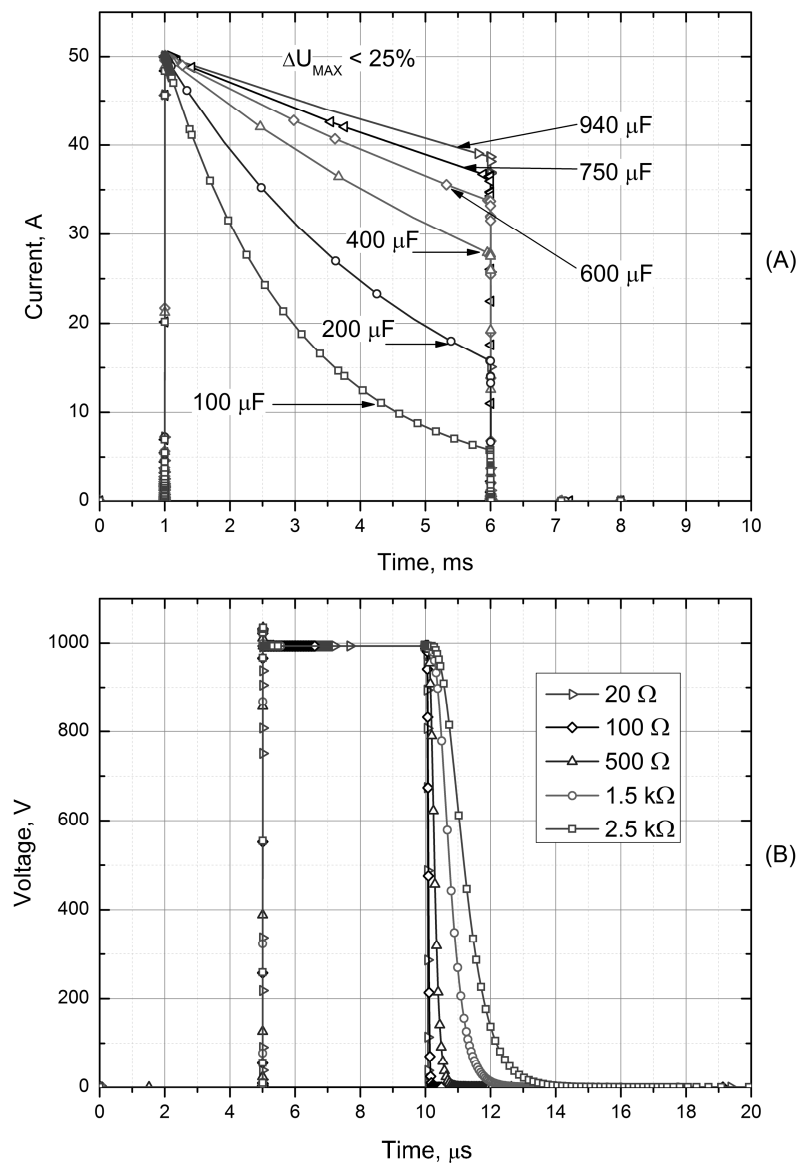

Fig.2. Performance of the electroporation system model with (A) different capacitor values; (B) different loads.

As it can be seen in Fig.2.(A) in order to acquire a square wave electrical pulse the high capacitor value above $750 \mu \mathrm{F}$ must be selected. Also currents up to $50 \mathrm{~A}$ and the charging voltage up to $1 \mathrm{kV}$ must be supported, which implies that the high power semiconductor switch must be used. Also due to the high currents and inevitable stray inductance in the circuit the snubber circuit should be implemented to prevent the overvoltage on the switch. In Fig.2.(B) the dependence of the pulse shape on the load of the system is presented. The influence of the transient processes is acceptable, however, as it was mentioned above, the implementation of a snubber diode is preferable. It can be noted that the pulse fall time depends on the load of the system resulting in up to $1.5 \mu$ s longer pulse with a highly resistive electrolyte $(2.5 \mathrm{k} \Omega)$. The delay can be removed if the load is shunted with a $100 \Omega$ resistor (Fig.2.(B)).

The model of the system showed a good performance and based on the results the MOSFET and pulse capacitors have been chosen: IXYS IXFN32N120 and two Epcos B25620B1487K101 $480 \mu \mathrm{F}$ capacitors, respectively. 


\section{DEVELOPED ELECTROPORATION SYSTEM}

Using the simulation results a compact electroporation system has been developed. The simplified schematic of the system is shown in Fig.3. The circuit is implemented using an adjustable power supply, divider resistor circuit, MOSFET with snubber diode, precise shunt resistance of $0.05 \Omega$ and a microcontroller. The microcontroller also controls the LCD and button type switches (S1-S5) that are used for input of the treatment parameters. Optical decoupling of the microcontroller circuit and the high power circuit has been implemented. Two 8-bit ADCs are implemented in the setup for the measurement of the charging voltage, output pulse parameters and the cuvette impedance change evaluation. The power supply is implemented using a flyback transformer voltage boost circuit. The capacitor array $\mathrm{C} 1$ has a total capacitance of $960 \mu \mathrm{F}$. The resistor R2 is used for the charged voltage measurement. The precise shunt resistor R4 is used for the output pulse waveform acquisition. The R3 is a current limiting resistor preventing the short circuit possibilities during the voltage breakdown in the cuvette. Also it is used as a reference for estimation of the voltage drop on the cuvette, allowing a straightforward recalculation of the cuvette impedance based on the measurement results from $\mathrm{ADC} 1$ and $\mathrm{ADC} 2$.

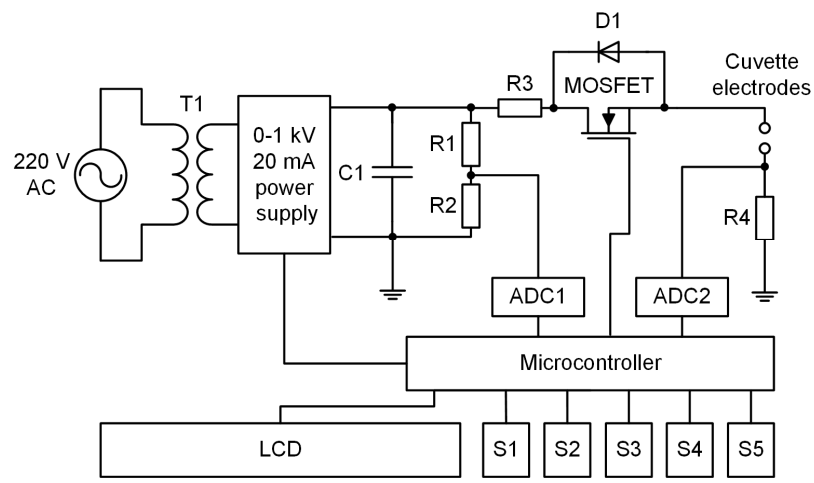

Fig.3. Simplified schematic of the electroporation system.

The electroporation system generated pulse shape dependence on various loads is presented in Fig.4. The $600 \mathrm{~V} 5 \mu \mathrm{s}$ pulse has been generated and the results have been compared to the simulation model.

As it can be seen in Fig.4. the experimental data is in an acceptable compliance with the simulation model. A $1.5 \mu \mathrm{s}$ $-1.8 \mu$ s delay is introduced when a more resistive load is used $(2.5 \mathrm{k} \Omega)$. The transient process in the beginning of the pulse results in the overvoltage in the range of $10 \%$ from the charged voltage, which is acceptable and complies with the simulation data. The capacitor discharge during single pulse meets the requirements of the electroporation system. The square-wave electroporation protocols in the $5 \mu \mathrm{s}-$ $5 \mathrm{~ms}$ duration range and the generation of the electric field up to $20 \mathrm{kV} / \mathrm{cm}$ with $0.5 \mathrm{~mm}$ gap cuvette are supported. Due to the high capacitance value $\mathrm{C} 1$ and the $20 \mathrm{~W}$ high voltage supply, the discharge of the capacitors does not limit the pulse burst electroporation protocols. The treatment is limited only by the thermal stress that will be experienced by the cells if a burst of high energy pulses is generated without temperature rise consideration. The repetitive frequency of the pulses can be controlled in the $0.1-100 \mathrm{~Hz}$ range.

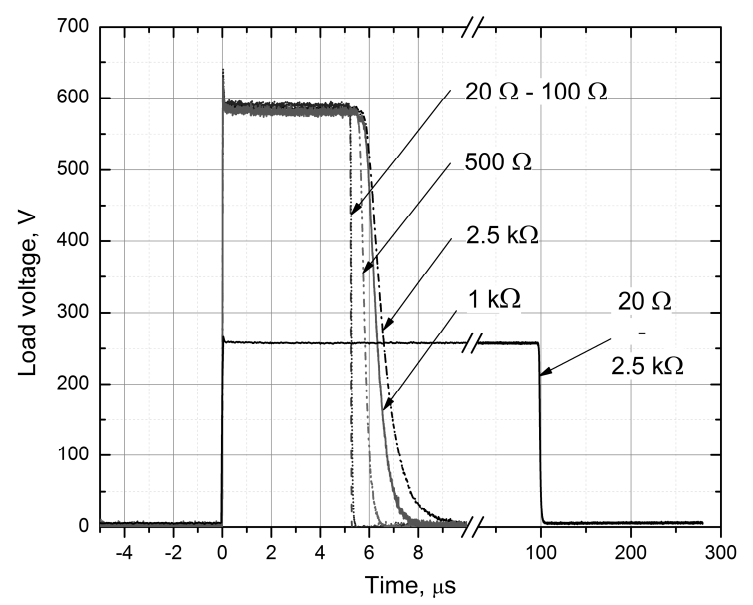

Fig.4. Generated pulses of the developed electroporation system.

The conductivity change measurement is performed during the pulse based on the readings on the ADC1 and ADC2. The current flowing through the cuvette is limited by the resistors R3, R4, the cuvette impedance and the resistance of the transmission line. At the same time the high precision resistors R3, R4 and the transmission line resistance are constant, which implies that the current in the transmission line will vary depending on the impedance change of the cell medium in the cuvette. Based on the voltage drop on the resistor R4, the pulsed current during electroporation is estimated. Straightforward recalculation to impedance change in the cuvette during the pulse is performed.

The photograph of the developed compact square wave electroporation system is shown in Fig.5. The size of the system is $21 \times 21 \times 25 \mathrm{~cm}$.

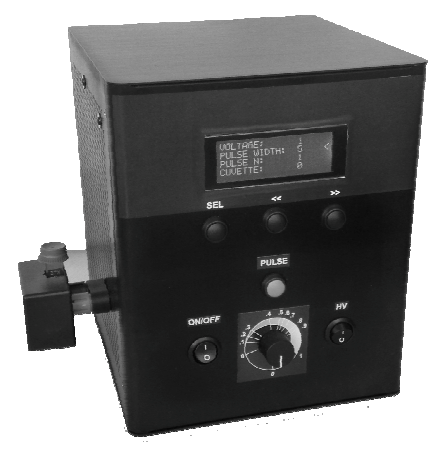

Fig.5. Photograph of the developed compact electroporation system.

The cuvette can be disconnected from the setup and complies with the sterility and the biomedical compatibility requirements for the electroporatoration. All standard commercial cuvettes can be used with the proposed setup.

The system is powered by a $220 \mathrm{~V}, 50 \mathrm{~Hz}$ line and has a total power consumption of $40 \mathrm{~W}$. 


\section{EXPERIMENT}

The system has been applied in the experiments with the freshwater mold Saprolegnia parasitica. This water mold can cause cellular necrosis or other epidermal damage to the fish or freshwater animals. The species have been isolated from the water samples collected in a freshwater pool (Vilnius, Verkiai park) using hemp seeds as a bait. The colonies on the hemp seeds formed after several days of incubation at room temperature. Later the fragments of hyphae and the reproductive spores were cut from colony edge, suspended in distilled water and used in the experiments. Samples of $80 \mu \mathrm{l}$ have been used for each experiment in $1 \mathrm{~mm}$ gap cuvette. The species were subjected to $400 \mathrm{~V}$ and $700 \mathrm{~V} 1 \mathrm{~ms}$ electrical pulses $(4 \mathrm{kV} / \mathrm{cm}$ and $7 \mathrm{kV} / \mathrm{cm}$, respectively). The repetitive frequency of $1 \mathrm{~Hz}$ was used, while the number of pulses has been altered from 1 to 20 . The electrical conductivity changes of the cell medium due to the electric field treatment are presented in Fig.6.

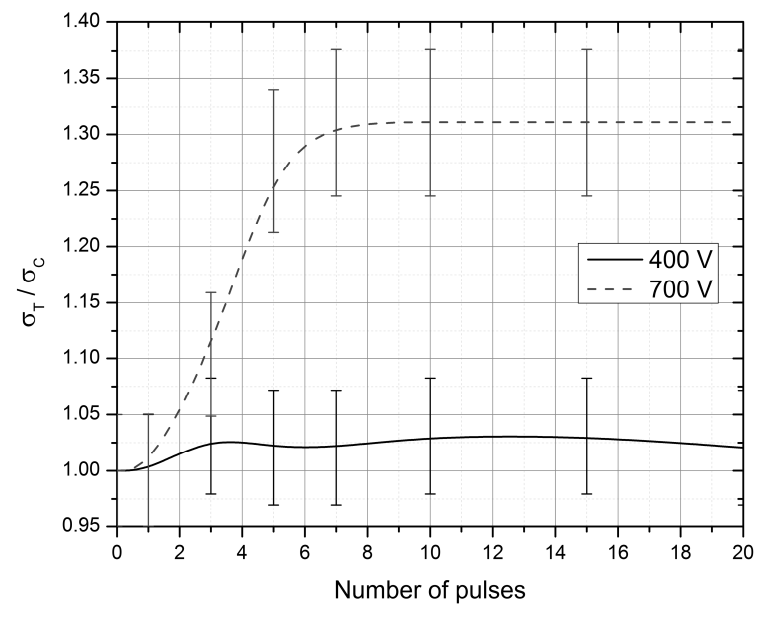

Fig.6. Cell medium electrical conductivity change during the treatment, where $\sigma_{\mathrm{T}}$ is the electrical conductivity during the treatment and $\sigma_{\mathrm{C}}$ is the electrical conductivity of the control sample.

As it can be seen in Fig.6. the electrical conductivity depends on the voltage applied. More than $30 \%$ increase has been observed when $10 \times 700 \mathrm{~V} 1 \mathrm{~ms}$ electrical pulses were generated. In the $400 \mathrm{~V}$ case the treatment intensity was not sufficient to cause significant changes. In order for the pores or irreversible damage in the membrane to appear (therefore the ion efflux), a voltage threshold potential (transmembrane potential) must be induced, which is proportional to the applied electric field. According to the experimental data the $7 \mathrm{kV} / \mathrm{cm}$ electric field is sufficient to stimulate ion efflux in the cells. However, the plateau region after $7 \times 700 \mathrm{~V}$ pulses indicates that the laceration and rapture of the Saprolegnia cells membrane (oogonial wall) occurred, when the cytoplasm could freely enter the cuvette electrolyte. The smaller oospores were mainly unaffected and higher electric field value was required. The microphotographs of the experimental samples have been taken for the morphological analysis of the species after the treatment and are shown in Fig.7.
According to the morphological analysis the membranes of the cells were not damaged when the $400 \mathrm{~V} 1 \mathrm{~ms}$ pulses were used, which corresponds with the conductivity change measurement experimental data. However, in the $700 \mathrm{~V}$ case (Fig.7.(B)) the membrane was heavily lacerated resulting in the release of the additional charge carriers into the electrolyte. The electrical conductivity of the medium is increased, respectively, which complies with the results presented in Fig. 6.

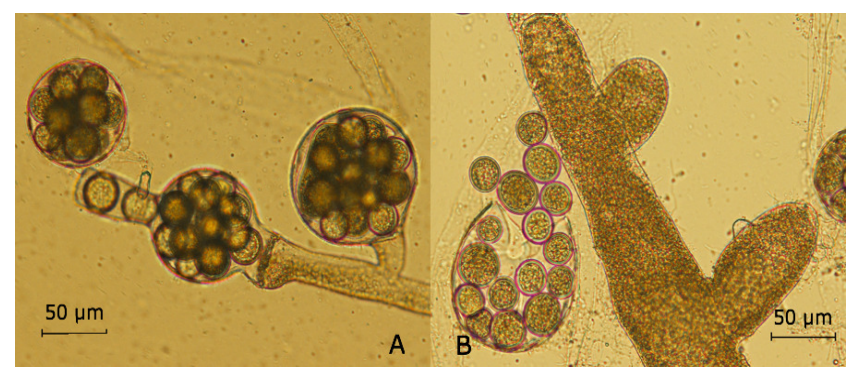

Fig.7. Microphotographs of the affected Saprolegnia parasitica samples: (A): $20 \times 1 \mathrm{~ms} 400 \mathrm{~V}$ pulses; (B): $20 \times 1 \mathrm{~ms} 700 \mathrm{~V}$ pulses.

It was concluded that the proposed system could be successfully applied in the electroporation experiments offering the extended analysis options and flexibility.

\section{CONCLUSIONS}

In this work the development of the universal electropermeabilization system is presented, which can be used with a variety of loads (pulse width independent peak current handling up to $50 \mathrm{~A}$ ).

The proposed setup is applicable for the majority of the currently known electroporation applications and the various types of cells. The capability of cuvette medium conductivity change measurement during each pulse allows the investigation of the dynamics of the electroporation process without the additional peripheral equipment such as LCR meters. The proposed system provides a solution to measure the conductivity change without the pulse synchronization issues.

The system has been successfully applied for electropermeabilization of pathogenic freshwater mold Saprolegnia parasitica. The proposed conductivity measurement module allowed predicting the effects caused by different electric field strengths. During $10 \times 700 \mathrm{~V} 1 \mathrm{~ms}$ pulsing more than $30 \%$ conductivity increase of the cell medium was observed. The morphological analysis showed laceration of the majority of the cells in the electrolyte. In the $10 \times 400 \mathrm{~V}$ $1 \mathrm{~ms}$ pulsing case the cells showed no signs of observable damage and also no changes in the electrical conductivity were observed.

We concluded that the proposed electroporation facility with conductivity change evaluation module could improve the quality of electroporation experiments by presenting the additional treatment effect evaluation parameter. The extent of the permeabilization process can be evaluated.

We think that the outlines for further development are to focus on the nanosecond electroporation range and the bipolar pulse generation support. These improvements will allow the investigation of the intracellular permeabilization procedures with a primarily non-thermal treatment, which is advantageous in this scientific area. 


\section{REFERENCES}

[1] Haberl, S., Miklavcic, D., Sersa, G., Frey, W., Rubinsky, B. (2013). Cell membrane electroporationPart 2: The applications. IEEE Electrical Insulation Magazine, 29 (1), 29-37.

[2] Cahill, K. (2010). Cell-penetrating peptides, electroporation and drug delivery. IET Systems Biology, 4 (6), 367-378.

[3] Tianyi, Z., Tatic-Lucic, S. (2012). On application of positive dielectrophoresis and microstructure confinement on multielectrode array with sensory applications. In IEEE Sensors 2012. IEEE, 1-4.

[4] Lei, U., Lo, Y.J. (2011). Review of the theory of generalised dielectrophoresis. IET Nanobiotechnology, 5 (3), 86-106.

[5] Potter, H., Heller, R. (2010). Transfection by electroporation. Current Protocols in Molecular Biology. DOI: 10.1002/0471142727.mb0903s62.

[6] Hung, M., Chang, Y. (2012). Single cell lysis and DNA extending using electroporation microfluidic device. BioChip Journal, 6 (1), 84-90.

[7] Hargrave, B., et al. (2013). Electroporation-mediated gene transfer directly to the swine heart. Gene Therapy, 20, 151-157.

[8] Pucinar, G., Krmelj, J., Rebersek, M., Napotnik, T.B, Miklavcic, D. (2011). Equivalent pulse parameters for electroporation. IEEE Transactions on Biomedical Engineering, 58 (11), 3279-3288.

[9] Sundararajan, R., et al. 2011. Effect of irreversible electroporation on cancer cells. In 2011 Annual Report Conference on Electrical Insulation and Dielectric Phenomena (CEIDP). IEEE, 164-167.

[10] Morshed, B.I., Shams, M., Mussivand, T. (2014). Investigation of low-voltage pulse parameters on electroporation and electrical lysis using a microfluidic device with interdigitated electrodes. IEEE Transactions on Biomedical Engineering, 61 (3), 871882.

[11] Khan, O.G.M., El-Hag, A.H. (2011). Biological cell electroporation using nanosecond electrical pulses. In 1st Middle East Conference on Biomedical Engineering (MECBME). IEEE, 28-31.

[12] Cima, L.F., Mir, L.M. (2004). Macroscopic characterization of cell electroporation in biological tissue based on electrical measurements. Applied Physics Letters, 85, 4520-4522.

[13] Davalos, R.V., Rubinsky, B., Otten, D.M. (2002). A feasibility study for electrical impedance tomography as a means to monitor tissue electroporation for molecular medicine. IEEE Transactions on Biomedical Engineering, 49 (4), 400-403.

[14] Pavlin, M., et al. (2005). Effect of cell electroporation on the conductivity of a cell suspension. Biophysical Journal, 88 (6), 4378-4390.

[15] Suzuki, D.O.H., Ramos, A., Ribeiro, M.C.M., Cazarolli, L.H. (2011). Theoretical and experimental analysis of electroporated membrane conductance in cell suspension. IEEE Transactions on Biomedical Engineering, 58 (12), 3310-3318.
[16] Kranjc, M., Bajd, F., Sersa, I., Miklavcic, D. (2011). Magnetic resonance electrical impedance tomography for monitoring electric field distribution during tissue electroporation. IEEE Transactions on Biomedical Engineering, 30 (10), 1771-1778.

[17] Davalos, R.V., Otten, D.M., Mir, L.M., Rubinsky, B. (2004). Electrical impedance tomography for imaging tissue electroporation. IEEE Transactions on Biomedical Engineering, 51 (5), 761-767.

[18] Granot, Y., Ivorra, A., Maor, E., Rubinsky, B. (2009). In vivo imaging of irreversible electroporation by means of electrical impedance tomography. Physics and Medicine in Biology, 54 (16), 4927-4943.

[19] Hjouj, M., Rubinsky, B. (2010). Magnetic resonance imaging characteristics of nonthermal irreversible electroporation in vegetable tissue. Journal of Membrane Biology, 236 (1), 137-146.

[20] Zhang, Y., et al. (2010). MR imaging to assess immediate response to irreversible electroporation for targeted ablation of liver tissues: Preclinical feasibility studies in a rodent model. Radiology, 256 (2), 424432.

[21] Sun, T., Morgan, H. (2010). Single-cell microfluidic impedance cytometry: A review. Microfluid Nanofluid, 8, 423-443.

[22] Das, D., Kamil, F.A., Biswas, K., Das, S. (2014). Evaluation of single cell electrical parameters from bioimpedance of cells suspension. RSC Advances, 4, 18178-18185.

[23] Weaver, J.C, Smith, K.C, Esser, A.T, Son, R.S, Gowrishankar, T.R. (2012). A brief overview of electroporation pulse strength-duration space: A region where additional effects are expected. Bioelectrochemistry, 87, 236-243.

[24] Zorec, B., Becker, S., Rebersek, M., Miklavcic, D., Pavselj, N. (2013). Skin electroporation for transdermal drug delivery: The influence of the order of different square wave electric pulses. International Journal of Pharmaceutics, 457 (1), 214-223.

[25] Charpentier, K.P, Wolf, F., Noble, L., Winn, B., Resnick, M., Dupuy, D.E. (2011). Irreversible electroporation of the liver and liver hilum in swine. $H P B, 13$ (3), 168-173.

[26] Miklavcic, D., Semrov, D., Mekid, H., Mir, L.M. (2000). A validated model of in vivo electric field distribution in tissues for electrochemotherapy and for DNA electrotransfer for gene therapy. Biochimica et Biophysica Acta, 1523, 73-83.

[27] Meir, A., Rubinsky, B. (2014). Electrical impedance tomographic imaging of a single cell electroporation. Biomedical Microdevices, 16 (3), 1387-2176.

[28] Polevaya, Y., Ermolina, I., Schlesinger, M., Ginzburg, B.Z., Feldman, Y. (1999). Time domain dielectric spectroscopy study of human cells II. Normal and malignant white blood cells. Biochimica et Biophysica Acta, 1419, 257-271.

[29] Chung, C., Waterfall, M., Pells, S., Menachery, A., Smith, S., Pethig, R. (2011). Dielectrophoretic characterization of mammalian cells above $100 \mathrm{MHz}$. Journal of Electrical Bioimpedance, 2, 64-71.

Received March 20, 2014. Accepted September 29, 2014. 